damit oft Frauen besonders betreffen. Von den bis 1992 geltenden Mindestentgelten bei der Rentenberechnung profitierten vor allem Frauen mit typischerweise niedrigerem Einkommen. Wenn die gesetzliche Altersrente im Umlagesystem auf der Grundlage des Dreigenerationenvertrages durch eine private kapitalgedeckte Altersrente ergänzt werden muss, so können sich das beispielsweise Alleinerziehende trotz staatlichem Zuschuss oft nicht oder nur in bescheidenem Umfang leisten, wodurch deren Altersarmut vorprogrammiert ist. Im Rentenversicherungsrecht sind jedoch bei der gesetzlichen Rente Strukturveränderungen nötig und möglich, die zu einer eigenständigen Alterssicherung von Frauen führen. Nirgends ist die Privatisierung und Individualisierung von sozialen Risiken so deutlich wie beim Risiko der Arbeitslosigkeit. Durch die drastische Verkürzung des Anspruchs auf die Versicherungsleis- tung Arbeitslosengeld greift bereits in der Regel nach zwölf Monaten Arbeitslosigkeit nur noch das Leistungssystem Hartz IV. Im Verhältnis zur „alten“ Arbeitslosenhilfe zeichnet sich dieses Leistungssystem durch eine verschärfte Anrechnung des Partnereinkommens aus, wodurch die Frauen ohne eigenständigen Leistungsanspruch auf die „Versorgerehe“ bzw. „Versorgerlebensgemeinschaft" verwiesen werden. Auch innerhalb des Leistungssystems Hartz IV gibt es diverse geschlechtsspezifische Verfestigungen (z. B. durch die Ausweitung von frauentypischen Minijobs als zumutbarer Beschäftigungsalternative) und durch die Art der Einkommensberechnung.

Die nachfolgenden Beiträge zu den einzelnen Leistungssystemen und zum Steuerrecht zeigen Handlungsbedarf und zwar auch nach 60 Jahren Grundgesetz und damit 60 Jahren verfassungsrechtlichem Gleichberechtigungsgebot.

\title{
Der Grundsatz der Gleichberechtigung von Frauen und Männern im Einkommensteuerrecht
}

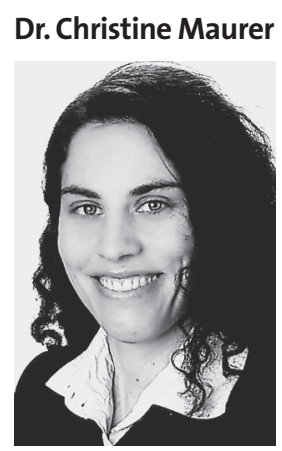

Mitglied der Kommission Recht der sozialen Sicherung, Familienlastenausgleich des djb; Rechtsanwältin, München

\section{Einleitung}

Auch im Einkommensteuerrecht gilt der Grundsatz der Gleichberechtigung von Frauen und Männern. Die besonderen Gleichheitssätze in Art. 3 Abs. 2 und 3 GG konkretisieren und verstärken den allgemeinen Gleichheitssatz in Art. 3 Abs. 1 GG. Sie konkretisieren daher auch die Steuergerechtigkeit im Hinblick auf geschlechtsbezogene Benachteiligungen und ziehen der Gestaltungsfreiheit des Gesetzgebers engere Grenzen. Dabei kommt dem Verbot der mittelbaren Diskriminierung besondere Bedeutung zu, weil das Steuerrecht unter anderem an geschlechtsbezogene Erwerbsmuster und Arbeitsmarktstrukturen sowie familiäre Rollenverteilungen anknüpft. Dadurch können Benachteiligungen verfestigt oder sogar verstärkt werden. Der Gesetzgeber ist daher gefordert, auf eine konsequente Umsetzung des Verbots der Benachteiligung im Einkommensteuerrecht hinzuwirken. Zentrale Kritikpunkte des djb sind nach wie vor das Ehegattensplitting, das Lohnsteuerverfahren für verheiratete Eheleute und die Absetzbarkeit von Kinderbetreuungskosten. Zudem zeigt sich, dass für eine Bewertung der realen Wirkungen der Besteuerung geschlechterdifferenzierte Statistiken auch im Steuerrecht notwendig sind.

\section{Reform der Ehebesteuerung}

Der djb fordert seit jeher die Abschaffung des Ehegattensplittings im Einkommensteuerrecht $(\mathbb{S} \mathbb{S}$ 26b, 32a Abs. 5 EStG). Die Kritik am Ehegatten- splitting betrifft vor allem die negativen Erwerbsanreize für Frauen ${ }^{1}$. Mit einer „zusätzlichen“ Berufstätigkeit der Ehefrau geht der Splittingvorteil verloren, so dass sich eine nicht nur geringfügige Berufstätigkeit erst lohnt, wenn der Splittingvorteil zumindest zurückverdient wird. Das Splittingvolumen fließt zu 93 Prozent in die alten Bundesländer. ${ }^{2}$ Diese Verteilung zeigt die Abhängigkeit des Splittingvorteils von geschlechtsbezogenen Erwerbsmustern und Arbeitsmarktstrukturen. In den neuen Bundesländern sind Frauen dabei nicht nur häufiger vollzeiterwerbstätig. Auch die Lohndifferenzen zwischen Frauen und Männern (gender pay gap) sind geringer. Gerade diese geringere Differenz senkt aber auch den Splittingvorteil. Das Ehegattensplitting konterkariert damit das Ziel, Lohndifferenzen abzubauen. Bei Arbeitnehmenden verstärkt die Lohnsteuerklasse $\mathrm{V}$ die Benachteiligung verheirateter Frauen durch die ungleiche Verteilung der Lohnsteuer zwischen den Eheleuten. Letztlich ist damit der vom Bundesverfassungsgericht bereits 1957 verbotene Edukationseffekt erhalten geblieben. ${ }^{3}$ Das Ehegatten-

1 Vgl. Hintergrundpapier 06-15 zur aktuellen Diskussion über eine Reform der Besteuerung von Ehe und Familie.

2 Bach, Stefan et al. (2003): Untersuchung zu den Wirkungen der gegenwärtigen Ehegattenbesteuerung auf Grundlage der fortgeschriebenen Einzeldaten der Einkommensteuerstatistik, Materialen des DIW Berlin, Nr. 27, S. 45 f.

3 Vgl. BVerfGE 6, 55 . 
splitting verfestigt überkommene Rollenverteilungen und trägt dazu bei, dass verheiratete Frauen erwerbslos bleiben bzw. ihre Erwerbstätigkeit aufgeben. Regelungen, die das Ehegattensplitting nur modifizieren oder auf die ganze Familie ausweiten, sind daher ebenso abzulehnen.

Die Zusammenveranlagung von Eheleuten und der Splittingtarif sind nach der Unterhaltsreform und der Einführung des Grundsatzes der Eigenverantwortung ( $\$ 1569$ BGB) endgültig nicht mehr zu rechtfertigen. Die jetzige Gesetzeslage führt dazu, dass während bestehender Ehe die Aufgabe der Berufstätigkeit der Frau gefördert und im Falle einer Scheidung die Aufnahme einer Erwerbstätigkeit gefordert wird. Um die Armut von Frauen nach einer Scheidung zu vermeiden, muss der Gesetzgeber darauf hinwirken, auch während bestehender Ehe Anreize für die Aufnahme bzw. Beibehaltung einer Erwerbstätigkeit zu schaffen oder zumindest negative Anreize abzuschaffen.

Die gleichstellungsorientierte Alternative zum Ehegattensplitting ist die Individualbesteuerung. Bestehende Unterhaltspflichten zwischen Eheleuten würden durch einen übertragbaren Grundfreibetrag berücksichtigt werden. Damit würden Unterhaltsgemeinschaften steuerrechtlich weitgehend gleich gestellt. Unterhaltsleistungen im Rahmen von Lebenspartnerschaften, Bedarfs- und nichtehelichen Lebensgemeinschaften können nämlich nur gemäß \33a EStG bis zu 7.680,00 Euro im Kalenderjahr vom Gesamtbetrag der Einkünfte abgezogen werden.

Die Reform der Ehebesteuerung wird auch von internationalen Organisationen wie der OECD und der UN (CEDAW) empfohlen. ${ }^{4}$ Erst im Februar 2009 forderte der Ausschuss für die Beseitigung der Diskriminierung der Frau bei den Vereinten Nationen Deutschland zum wiederholten Mal auf, die aktuellen gesetzlichen Vorschriften zur Besteuerung von Ehepaaren („Splitting“) sowie deren Auswirkungen auf das Fortbestehen stereotyper Erwartungen an verheiratete Frauen zu überprüfen. ${ }^{5}$

Die für 2010 vorgesehene Einführung des sogenannten Faktorverfahrens im Lohnsteuerverfahren kann den Übergang zu einer Individualbesteuerung nicht ersetzen. Das Faktorverfahren ermöglicht zwar eine gerechte Verteilung der Lohnsteuer bei verheirateten Arbeitnehmenden. Zum einen bleiben aber das Ehegattensplitting und damit auch dessen negative Erwerbsanreize erhalten, zum anderen wird das Faktorverfahren lediglich als Option neben der Lohnsteuerklassenkombination III/V eingeführt. Es bleibt abzuwarten, ob sich das Faktorverfahren als gerechtere Lohnsteuerverteilung zwischen den Eheleuten durchsetzt. ${ }^{6}$

\section{Absetzbarkeit von Kinderbetreuungskosten als Werbungskosten/Betriebsausgaben}

Erwerbsbedingt veranlasste Kinderbetreuungskosten sollten als Werbungskosten vom ersten Euro an steuerlich absetzbar sein. Nach der derzeitigen Regelung in $\mathbb{S} \mathbb{S}$ Abs. 5, 9c EStG sind erwerbsbedingte Kinderbetreuungskosten nur „wie“ Werbungskosten/Betriebsausgaben absetzbar, weil Aufwendungen für die Kinderbetreuung immer noch der Privatsphäre zugeordnet werden. ${ }^{7}$ Dies obwohl Kinderbetreuung bei Erwerbstätigkeit beider Eltern zwingend notwendig ist. Solche Aufwendungen sind Werbungskosten/Betriebsausgaben, deren Absetzbarkeit das objektive Nettoprinzip fordert. Infolgedessen bedarf es einer realitätsgerechten Berücksichtigung von erwerbsbedingten Kinderbetreuungskosten. Unabhängig davon obliegt es dem Gesetzgeber freilich auch, durch den Ausbau von Kinderbetreuungseinrichtungen und eine bedarfsabhängige, zielgerichtete Förderung von Kindern zunächst einmal Sorge dafür zu tragen, dass Eltern überhaupt die Wahl zwischen Eigenbetreuung der Kinder und beiderseitiger Berufstätigkeit haben.

\section{Erhebung von Daten zu Wirkungen der Besteue- rung}

Eines der grundlegenden Probleme im Steuerrecht sind fehlende Statistiken, die nach Geschlecht und anderen Benachteiligungsdimensionen differenzieren. Es fehlt bereits an geschlechterdifferenzierten Statistiken, die in einem Vergleich von Brutto- und Nettoeinkommen zeigen, ob die Besteuerung den Gender Pay Gap verstärkt oder mindert. ${ }^{8}$ Auch sind mangels entsprechender Daten die Wirkungen des Steuerrechts auf die Einkommens- und Beschäftigungssituation von Migrantinnen und Migranten

4 www.oecd.org/dataoecd/27/35/40382005.pdf, S. 13; BT-Drs. 15/105, S. 11.

5 Schlussempfehlungen zum sechsten Staatenbericht Deutschlands: CEDAW/C/DEU/CO/6 v. 9.2.2009.

6 Stellungnahme 08-15 zum Entwurf der Bundesregierung zum Jahressteuergesetz 2009 (JStG 2009) (BTDrs. 16/10189), Einführung eines optionalen „Faktorverfahrens" für die Lohnsteuer bei Ehegatten, § 39 f EStG - neu-.

7 Stellungnahme 06-03 zum Gesetzentwurf von CDU/ CSU und SPD zur steuerlichen Förderung von Wachstum und Beschäftigung vom 14.2.2006 BT-Drucks. $16 / 643$.

8 Österreich hat beispielsweise nicht nur die Wirkungen der Einkommensbesteuerung insgesamt, sondern auch einzelne Steuerentlastungen untersucht. Die Auswertung zeigt, dass Steuerentlastungen fast überwiegend Männern zu Gute kommen, die Steuerentlastungen nicht nur der Zahl, sondern auch der Höhe nach überproportional nutzen, vgl. Einhaus, Arnd et al. (2006): Ist die Einkommensbesteuerung geschlechtsneutral?, www.kfunigraz.ac.at/akglwww/archiv/ Steuerstudie.pdf.

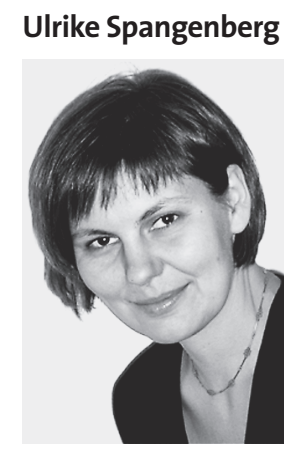

Mitglied der Kommission Recht der sozialen Sicherung, Familienlastenausgleich des djb; Juristin, Berlin 
im Vergleich zu Personen ohne Migrationshintergrund für Deutschland bislang schwer zu ermitteln. Untersuchungen für Deutschland zeigen beispielsweise, dass haushaltsnahe Dienstleistungen oder Pflegeleistungen häufig von Frauen mit Migrationshintergrund angeboten werden. ${ }^{9}$ Sowohl das Verbot der mittelbaren Diskriminierung als auch das Gleichstellungsgebot fordern die Erhebung und Auswertung entsprechender Daten. Die übliche Darstellung der Auswirkungen von Steuernormen nur für Alleinverdienstehen genügt dieser Anforderung nicht. Der Gesetzgeber ist verpflichtet, faktische Diskriminierungen, die sich als Folge einer Regelung ergeben, soweit wie möglich zu vermeiden. Als Mindeststandard setzt ein effektiver Grundrechtsschutz voraus, dass der Gesetzgeber ausreichende Vorkehrungen für einen angemessenen und wirksamen Schutz trifft. Diese Vorkehrungen müssen auf sorgfältigen Tatsachenermittlungen und vertretbaren Einschätzungen beruhen. Ein geeignetes Instrument dafür ist eine geschlechterdifferenzierte Folgenabschätzung, wie sie auch $\mathbb{} 2$ der Gemeinsamen Geschäftsordnung der Bundesministerien (GGO) vorsieht. Allerdings genügt der in Gesetzentwürfen übliche Hinweis auf die fehlende gleichstellungsrechtliche Relevanz gesetzlicher Regelungen nicht. Es ist Aufgabe des Parlaments, die Verpflichtung der Bundesregierung aus $\mathbb{} 2$ GGO zu prüfen und differenzierte Folgenabschätzungen einzufordern.

\section{Zusammenfassung}

Der Staat ist auch bei der Einkommensbesteuerung gehalten, die tatsächliche Durchsetzung der Gleichberechtigung von Frauen und Männern zu fördern und auf die Beseitigung bestehender Nachteile hinzuwirken. Regelungen wie das Ehegattensplitting, die sich in typisierender Form an der Einverdienst-Ehe ausrichten und damit in Widerspruch zu diesem Gebot stehen, sind abzuschaffen. Ferner sollten sie selbstverständlich gemäß dem Grundsatz der Einheitlichkeit der Rechtsordnung auch in Einklang stehen mit anderen Rechtsgebieten, insbesondere dem Unterhalts- und Sozialrecht.

Die angesprochenen Themen werden auch in Zukunft vom djb diskutiert. Weitere Themen sind unter anderem die Besteuerung von Minijobs und die dadurch ausgelösten Erwerbsanreize für Beschäftigungen ohne eine längerfristige soziale Absicherung sowie die Bewertung von umsatzsteuer(teil)befreiten Leistungen aus gleichstellungspolitischer Sicht.

9 Vgl. Lutz, Helma: Weltmarkt Haushaltsarbeit - ein blinder Fleck in der Gleichstellungspolitik. Gender Lecture am 9.2.2009 im Rahmen des Schwerpunktthemas „Wert von Arbeiten“ http://db.genderkompetenz.info/deu/archive/events/ gendlectkompetenz/2009_02_09_hu/.

\section{Gesetzliche Krankenversicherung}

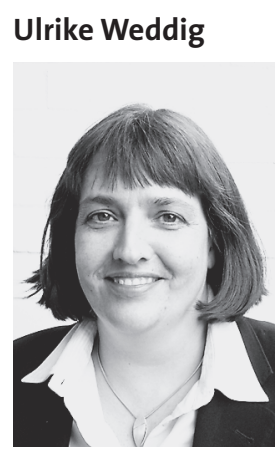

Mitglied der Kommission Recht der sozialen Sicherung, Familienlastenausgleich des djb; Richterin am Landessozialgericht, Celle
Die Diskussion um die Reform des deutschen Gesundheitssystems, insbesondere der Gesetzlichen Krankenversicherung, bleibt brandaktuell. Die Änderungen durch das Gesetz zur Stärkung des Wettbewerbs in der Gesetzlichen Krankenversicherung (GKV-Wettbewerbsstärkungsgesetz - GKV-WSG) vom 26. März 2007 (BGBl. I S. 378) haben bei weitem nicht alle Probleme gelöst, sondern viele offene Fragen hinterlassen.

Die bis zur vorgezogenen Bundestagswahl 2005 geführte Debatte über zwei sich als scheinbar unvereinbar gegenüberstehende Reformmodelle für die GKV - Kopfpauschale/Gesundheitsprämie oder Bürgerversicherung - ist nun gemündet in das Modell der „Gesundheitsversicherung“ mit dem neuen Instrument des Gesundheitsfonds, der seit dem 1. Januar 2009 Realität geworden ist.

Die Arbeitsgruppe Gesetzliche Krankenversicherung (GKV) der Kommission Soziale Sicherung hat die Einführung des Gesundheitsfonds kritisch begleitet.

Schon im Vorfeld wurde seitens des djb darauf aufmerksam gemacht, dass
- die zunehmende „Verbetriebswirtschaftlichung“ die strukturellen Probleme der GKV nicht lösen kann,

- die GKV vielmehr zunächst auf eine solide Einnahmebasis gestellt werden muss,

- Strukturreformen erforderlich sind, die dazu beitragen, Unter-, Über- und Fehlversorgungen zu vermeiden, den Gedanken der Prävention zu stärken und eine geschlechtergerechtere Versorgung zu ermöglichen bzw. sicherzustellen.

Als übergeordnetes Problem ist hier die Betrachtung der GKV unter zunehmend betriebswirtschaftlichen Gesichtspunkten zu nennen. Es steigt der Druck, betriebswirtschaftliche Kriterien über den sozialen Absicherungs- und Versorgungsauftrag zu stellen. Damit geht die Gefahr einher, die Krankenversicherung als Teil der Daseinsvorsorge zur Disposition zu stellen. Natürlich können betriebswirtschaftliche Aspekte, wie zum Beispiel Wirtschaftlichkeit, nicht ausgeblendet werden. Sie dürfen aber nicht dazu führen, das originäre Leistungsangebot des öffentlichen Gesundheitswesens 